\section{Ankle edema after administra- tion of selective serotonin reuptake inhibitors}

\author{
Konstantinos Kontoangelos, \\ Marina Ecomomou, \\ Charalambos Papageorgiou
}

First Department of Psychiatry, Eginition Hospital, Athens; University Mental Health Research Institute, Athens, Greece

\begin{abstract}
Clinical manifestations of drug-induced skin reactions include a wide range of symptoms, from mild drug-induced exanthemas to dangerous and life-threatening generalized systematic reactions. Druginduced skin reactions to psychotropic medication are usually associated with antiepileptic drugs. However, a significant role can be assigned to selective serotonin reuptake inhibitors. We report a case of a female patient, who after approximately one month therapy with escitalopram developed a bilateral ankle edema, which resolved completely within the first week following its discontinuation. Although serious complications are rare, clinicians should be aware of severe skin complications in patients treated with antidepressants, which necessitate careful clinical monitoring and management. Individualization of pharmacotherapy is crucial, together with regular evaluation of safety and tolerance of the treatment.
\end{abstract}

\section{Introduction}

Skin is a biological barrier that protects the internal milieu from the external environment, and its subjected to variable interactions with solar, thermal, radiant and mechanical energy and these environmental factors may participate in the pathogenesis of skin diseases. ${ }^{1}$ Skin capacity to recognize and discriminate signals is probably influenced by skin immune and pigmentary systems; however the high level barrier of the skin is probably under the influence of a local endocrine system. ${ }^{2}$ Serotonin is known to have many roles in skin for example proedema vasolidatory proinflammatory and pruritogenic. Receptors for serotonin are expressed in keratinocytes melanocytes, and fibroblasts and these mediate phenotypic actions on cellular proliferation and differentiation. ${ }^{3}$ Serotonin (5-hydroxytrypta- mine) is the product of multistep metabolic pathway: it starts with the ratelimiting step of L-tryptophan hydroxylation by tryptophan hydroxylase (TPH), which requires $6 \mathrm{BH} 4$ as cofactor. ${ }^{4}$ Serotonin is predominantly synthesized by intestinal enterochromafin cells, with smaller quantities produced by the CNS, rectum (cylindrical epithelium), bronchial cells (epithelium), thyroid parafollicular cells, ovaries, thymus, pancreas breast, and skin. ${ }^{5}$ We report a case of a female patient who after approximately one month therapy with escitalopram (ESC) developed a bilateral ankle edema, which resolved completely within the first week following its discontinuation.

\section{Case Report}

This is a case of a 52-year-old female, with a recent history of panic attack and depression, according to DSM-IV-TR criteria, triggered by a recent retirement. Laboratory and clinical evaluations during the last years had never revealed any pathological findings. At her referral to our department (community mental health service), the patient was suffering from depressed mood most of the day, fatigue, diminished ability to think or concentrate, insomnia, feelings of worthlessness and periods of sweating, chest pain, dizziness and fear of losing control. ESC was administered with an initial dose of $10 \mathrm{mg}$ once a day for a week, and thereafter to $20 \mathrm{mg}$ per day. Three weeks later the patient's depressive symptoms improved significantly; however she complained about a swelling in both of her feet and she could hardly wear her shoes. The clinical examination revealed a bilateral ankle edema.

Clinical and laboratory evaluations (electrocardiogram, ultrasonography, blood tests, albumins, renal and thyroid function tests, serum electrolytes) did not reveal any pathological findings. There was also no history of arterial hypertension, and after repeated measurements her arterial pressure was within normal range. The patient's edema resolved completely within ten days after escitalopram discontinuation with no reappearing.

\section{Discussion}

In the literature there are several references concerning SSRI administration and skin pathology. Welsh et al. described first an incidence of paroxetine induced urticarial vasculitis, which is chronic disorder marked by recurrent episodes of erythema-
Correspondence: Konstantinos Kontoangelos, Athens University Medical School, 1st Department of Psychiatry, Eginition Hospital, 74 Vas Sofias Avenue, 11528, Athens, Greece. Tel: +30-210-6170071 - Fax: +30-210-7242020 E-mail: kontange@hol.gr

Key words: escitalopram, edema, depression, serotonin, skin, cutaneous effects.

Contributions: KK participated in the acquisition of data, analysis and interpretation of data, and wrote the first draft of the manuscript; ME participated in the study concept and design, acquisition and interpretation of the data, and drafting and critical revision of the manuscript and had the overall supervision and corrected the final draft.

Conflict of interest: the authors declare no potential conflict of interest.

Funding: none.

Received for publication: 19 August 2017. Revision received: 20 October 2017. Accepted for publication: 26 October 2017.

This work is licensed under a Creative Commons Attribution-NonCommercial 4.0 International License (CC BY-NC 4.0).

(C) Copyright K. Kontoangelos et al., 2018

Licensee PAGEPress, Italy

Mental Illness 2018; 10:7364

doi:10.4081/mi.2018.7364

tous, indurated wheals that histologically manifest the features of leukocytoclastic vasculitis. 6 Vermeer et al. described two case reports in two patients with mycosis fungoides who had an exacerbation of their disease shortly after starting fluoxetine. Fluoxetine is not a carcinogenic agent but it may have tumor growth promoting and immune-modulating properties. ${ }^{7}$

Kraskowa et al. has substantiated an increased risk of bleeding events probably caused by blockade of serotonin reupatake in platelets and subsequent platelet dysfunction. Other cutaneous effects of the most commonly used antidepressant medication with SSRI are also petechiae, ecchymosed, spontaneous bruising, acneiform eruption, leukocytoclastic vasculitis, urticaria, angioedema, erythema nodosum, erythema multiforme, alopecia, photoxic reactions, photoallergic reactions, acute generalized exanthematous pustulosis. ${ }^{8}$ Angioedema has been specifically associated with the use of paroxetine, bupropion and trazodone and it is believed to be type I hypersensitivity reactions. Angioedema affects the face, tongue and extremities and involves a larger edematous area including the dermis and 
subcutaneous tissue. ${ }^{9}$

Masdrakis et al. from the same department reported a similar case of a 69-yearold female depressed patient who, after approximately 1 month of therapy with ESC, progressively titrated up to 30 $\mathrm{mg}$ /day, developed a bilateral ankle edema, which resolved completely within the first week following its discontinuation. ${ }^{10}$ As a possible pathopsysicological mechanism lipid-soluble psychotropics are often used to treat skin diseases with psychosomatic indications and several lipid-soluble psychotropic drugs have been examined for their ability to inhibit protein kinase C (PKC)-catalyzed phosphorylation of exogenous substrates and endogenous skin proteins. Phosphorylation of three discrete skin protein substrates at 64, 42 and $28 \mathrm{kDa}$ and a group crowded together at $15-18 \mathrm{kDa}$ was prevented by the antidepressants/antipsychotics. Inhibition w as more pronounced in a phospholipid (PL) dependent system, but both drug-PL and drug-PKC interactions seem to be important in the mechanism of action of these drugs. In addition to the tricyclic nucleus, the propanamine side chain or its N-methyl form may influence the interaction of these drugs with PKC and its substrate(s). Chlorpromazine, imipramine, fluoxetine, doxepin, amitriptyline and hydroxyzine used in the practice of dermatology may exert their therapeutic effects by modulating skin PKC activity. ${ }^{11}$ The relation between cutaneous pseydolymphopmas and antidepressant therapy in eight patients treated with fluoxetine hydrochloride revealed that cutaneous pseudolymphomas are associated with antidepressant (AD) therapy, possibly reflecting perturbation of lymphoid function. Concomitant therapy with agents that have additive or synergistic immunomodulatory effects or an immunedysregulating systemic disease may increase patient's susceptibility to developing atypical cutaneous lymphoid hyperplasia while the patient is receiving AD therapy. ${ }^{12}$

\section{Conclusions}

Bilateral ankle edema constitutes a rare side effect during therapy with ESC. There is further need for close therapeutic monitoring in everyday clinical practice and before the prescription of any antidepressant agent, one should be aware of the specific guidelines, side-effect profile, drugdrug interactions and most current indications.

\section{References}

1. Slominski A, Wortsman J. Neuroendocrinology of the skin. Endocr Rev 2000;21:457-87.

2. Slominski A, Wortsman J, Pisarchik A, et al. Cutaneous expression of corticotropin-releasing hormone $(\mathrm{CRH})$, urocortin, and CRH receptors. FASEB J 2001;15:1678-93.

3. Slominski A, Wortsman J, Tobin DJ. The cutaneous serotoninergic/mela- toninergic system securing a place under the sun. Faseb J 2005;19:176-9.

4. Mockus SM, Vrana KE. Advances in the molecular characterization of tryptophan hydroxylase J Mol Neurosci 1998;10:163-79.

5. Slomiski A, Pisatchik A, Semak I et al. Serotonergic and melatoninergic systems are fully expressed in human skin. Faseb J 2002;16:896-8.

6. Welsh JP, Cusack CA, Ko C. Urticarial vasculitis secondary to paroxetine. J Drugs Dermatol 2006;5:1212-4.

7. Vermeer MH, Willemze R. Is mycosis fungoides exacerbated by fluoxetine? J Am Acad Dermatol 1996;35:635-6.

8. Krasowka D, Szymanek M, Schwartz R, Myslinski W. Cutaneous effects of the most commonly used antidepressant medication, the selective serotonin reuptake inhibitors. J Am Acad Dermatol 2007;56:848-53.

9. Warnock JK, Morris DW. Adverse cutaneous reactions to antidepressants. Am J Clin Dermatol 2002:3;329-39.

10. Masdrakis VG, Oulis P, Kouzoupis AV, et al. Bilateral ankle edema in a patient taking escitalopram. World J Biol Psychiatry 2009;10:939-41.

11. Vaitla R, Roshani P, Holian O, et al. Inhibition of skin protein kinase $\mathrm{C}$ by psychotropic drugs. Skin Pharmacol 1997;10:191-9.

12. Crowson AN, Magro CM. Antidepressant therapy. A possible cause of atypical cutaneous lymphoid hyperplasia. Arch Dermatol 1995;131:925-9. 